\title{
Detection Of Shiga-Toxin Producing E.coli (STEC), Enteropathogenic E.coli (EPEC) And Enterotoxigenic E.coli (ETEC) From Animals By M-PCR
}

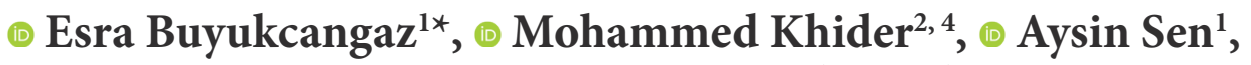 \\ (1) Murat Cengiz ${ }^{3}$, ๑) Songül Sonal ${ }^{3}$
}

\author{
1Bursa Uludag University, Faculty of Veterinary Medicine, Department of Microbiology, 16059, Bursa, Turkey \\ 2BursaUludag University, Institute of Health Sciences, 16059, Bursa, Turkey \\ 3BursaUludag University, Faculty of Veterinary Medicine, Department of Pharmacology \& Toxicology, 16059, Bursa, Turkey. \\ 4 University of Bahri, College of Veterinary Medicine, Department of Microbiology, Khartoum, Sudan.
}

Received 26.09.2019 Accepted 10.11.2019

\begin{abstract}
This research investigated the presence of virulence genes encoding F41, K99, eae, Stx1, Stx2 and STa and the antimicrobial resistance of animal Escherichia coli (E. coli) isolates. Clinical isolates (n:233) were evaluated from fecal samples of cattle, sheep, goats, horses, cats and dogs collected between the years of 2010 to 2015 from Turkey. Enterohaemorrahic E.coli (EHEC) O157:H7 was detected by using cefixime tellurite sorbitol MacConkey agar (CT-SMAC) and Wellcollex E. coli (Remel ${ }^{\circledR}$ ). The Kirby-Bauer disc diffusion test was performed to detect the resistance pattern of the isolates to ampicillin, Amoxycilin/clavulanic acid, enrofloxacin, ceftiofur, trimethoprim/sulfamethoxazole and tetracycline. The results showed that $40 \%$ of the ruminant isolates were identified as Shiga-toxin producing Escherichia coli (STEC). Enterotoxigenic E. coli (ETEC) was detected in samples from cattle (0.9\%) and sheep (12\%). Enteropathogenic E.coli (EPEC) was detected in samples from cattle (0.9\%) and dogs (11.4\%). EHEC O157:H7 was not detected any of the isolate. Among all E.coli isolates that carried at least one virulence gene, 8 (19\%) were resistant to more than three antimicrobials, 7 (16.7\%) were resistant to at least one antimicrobial and 27 (64.3\%) were susceptible to all antimicrobials.
\end{abstract}

Keywords: Antimicrobial susceptibility, E. coli, PCR, STEC, EPEC, ETEC

\section{Introduction}

E.coli occur naturally in the lower part of the intestine microbiota of humans and warm-blooded animals. Most strains of E.coli, do not cause disease in healty persons, however there are specific pathogenic groups, whose members are capable of causing disease in humans and animals. Pathogenic E.coli strains are broadly grouped into two categories, extraintestinal pathogenic E.coli and intestinal or diarrhagenic E.coli depending on whether they cause disease outside or within the intestinal tract. ${ }^{1}$ There are at least five categories of recognized diarrhagenic E.coli, Shiga toxin-producing E.coli (STEC) or verotoxigenic E. coli (VTEC); which includes subset of strain referred to as enterohemorragic E.coli for their ability to cause bloody diarrhea and haemorragic colitis, enterotoxigenic E. coli (ETEC), enteropathogenic E.coli (EPEC), enteroaggregative E.coli (EAEC) and enteroinvasive E.coli (EIEC). Enterohemorrhagic E.coli (EHEC) strains comprise a subgroup under STEC that cause bloody diarrhea.

\footnotetext{
* Corresponding author: Esra Buyukcangaz, kocakaya@uludag.edu.tr
} 
EHEC serotype O157:H7 is the most common serotype worldwide. ${ }^{1-4}$

EPEC strains are enteric pathogens that lead to diarrheal illness; they produce attaching and effacing (A/E) lesions and intimate attachment of EPEC to epithelial cells in the gut mucosa of human or animal hosts. ${ }^{5,6}$ In humans and young farm animals, ETEC infections can cause watery, non-bloody diarrhea. ${ }^{3}$ ETEC possess two virulence factors: fimbriae (pili) and enterotoxins. F5 (K99) and/or F41 fimbriae mediate adherence to the ileum, while thermolabile (LT) and thermostabile (STa and STb) enterotoxins stimulate a secretory response by intestinal crypt cells. ${ }^{7}$

Recently, antimicrobial resistance in E.coli has increased remarkably. ${ }^{8}$ In addition, commensal and pathogenic E.coli isolates can be important reservoirs for antimicrobial resistance determinants, which may be transferred via transmissible plasmids within species. ${ }^{9}$

This article presents the results of the targeted diarrheagenic E.coli virulence genes by multiplex PCR and the resistance pattern of veterinary importance antimicrobials in virulent and avirulent E.coli isolates from domestic animals in Turkey.

\section{Materials and Methods}

The study was conducted on animals from Bursa city and province, regardless of health status, that were brought to Uludag University Animal Hospital in the period between 2010 and 2015. A total of 233 E.coli isolates were recovered from fecal samples of cattle (n:111), sheep (n:25), goats (n:45), horses (n:4), cats (n:13) and dogs (n:35).

E.coli isolates from a variety of animals were screened for the virulence genes encoding eae, STa, Stx1, Stx2, F41 and
K99 with mPCR according to the method described by Franck et al. 10 with certain modifications (Table 1).

All primers' sequences used in this study are shown in Table 1 . The amplification was carried out in a Techne ${ }^{\circledast}$ TC$3000 \mathrm{G}$ gradient thermal cycler (Bibby Scientific, UK) with the following steps: initial denaturation at $98^{\circ} \mathrm{C}$ for $5 \mathrm{~min}$; 25 cycles at $98^{\circ} \mathrm{C}$ for $30 \mathrm{~s}$, annealing at $55^{\circ} \mathrm{C}$ for $30 \mathrm{~s}$ and extension at $70^{\circ} \mathrm{C}$ for $1 \mathrm{~min}$; final extension at $70^{\circ} \mathrm{C}$ for 10 min. The PCR products were electrophoresed in $1.4 \%$ agarose gel (BIO-ROC, UPL Diagnostic Mainz - Germany) for $100 \mathrm{~min}$ at $80 \mathrm{~V}$ and visualized under UV light (VilberLoumat $^{\oplus}$ - Quantum ST4- France). E.coli O157:H7 (ATCC 35150) and E.coli 0101(ATCC PTA-5951) were used for the reference strains. E.coli (ATCC 25922) and sterile distilled water were used for the negative control.

The Stx1, Stx2, eae positive isolates were inoculated onto cefixime tellurite (Oxoid ${ }^{\circledR}$ - SR0172) sorbitol MacConkey agar (CT-SMAC) (Oxoid ${ }^{\circledast}$ - CM0813) followed by incubation at $37^{\circ} \mathrm{C}$ for $18-24 \mathrm{~h}$ for the detection of EHEC O157:H7. The presence of sorbitol negative (colorless) colonies were checked. Non-sorbitol-fermenting colonies from the CT-SMAC agar plates were examined by the latex agglutination test Wellcollex E.coli (Remel $^{\oplus}$ Europe Ltd. UK). Specific antisera was used to the determination of E.coli $\mathrm{O} 157$-H7 as described by the manufacturer.

Virulent and avirulent E.coli isolates (n:233) were detected and evaluated with the Kirby-Bauer disc diffusion test for ENR (Oxoid $\left.{ }^{\circledast}, 5 \mu \mathrm{g}\right)$, AMP (Oxoid $\left.{ }^{\circledR}, 10 \mu \mathrm{g}\right)$, TE (Oxoid ${ }^{\circledR}, 30$ $\mu \mathrm{g})$, SXT (Oxoid $\left.{ }^{\circledR}, 25 \mu \mathrm{g}\right), \mathrm{CTF}\left(\right.$ Oxoid $\left.^{\circledR}, 30 \mu \mathrm{g}\right)$ and AMC (Oxoid $^{\circledast}, 30 \mu \mathrm{g}$ ) according to EUCAST Version 6.0 (201601-01) directions. ${ }^{11}$

Table 1: Primers used in this study for the multiplex PCR (Franck et al. 1998)

\begin{tabular}{|c|l|c|}
\hline Stx1 & $\begin{array}{l}\text { (F) TTCGCTCTGCAATAGGTA } \\
\text { (R) TTCCCCAGTTCAATGTAAGAT }\end{array}$ & \\
\hline Stx2 & $\begin{array}{l}\text { (F) GTGCCTGTTACTGGGTTTTTCTTC } \\
\text { (R) AGGGGTCGATATCTCTGTCC }\end{array}$ & 118 \\
\hline Intimin & $\begin{array}{l}\text { (F) ATATCCGTTTTAATGGCTATCT } \\
\text { (R) AATCTTCTGCGTACTGTGTTCA }\end{array}$ & 425 \\
\hline F41 & $\begin{array}{l}\text { (F) GCATCAGCGGCAGTATCT } \\
\text { (R) GTCCCTAGCTCAGTATTATCACCT }\end{array}$ & 380 \\
\hline K99 & (F) TATTATCTTAGGTGGTATGG & 314 \\
& (R) GGTATCCTTTAGCAGCAGTATTTC & 190 \\
\hline STa & (F) GCTAATGTTGGCAATTTTTATTTCTGTA & \\
\hline
\end{tabular}




\section{Results}

Results showed that virulence genes indicating the presence of DEC were detected in samples from $9(8.1 \%)$ cattle, 13 (52\%) sheep, 15 (33.3\%) goats, and 4 (11.4\%) dogs. Virulence genes were detected at the following rates: Stx1 26 (11.1\%), Stx2 17 (7.3\%), eae 12 (5.2\%) and STa 4 (1.7\%). All horse and cat isolates were negative for all genes. Virulence genes indicating the presence of DEC were detected in this study in the following percentages: STEC in $6.3 \%, 40 \%$ and $33.3 \%$ of isolates from cattle, sheep and goats, respectively; ETEC in $0.9 \%$ and $12 \%$ of isolates from cattle and sheep, respectively; and EPEC in $0.9 \%$ and $11.4 \%$ of isolates from cattle and dogs, respectively (Table 2 ).

In total, 8 cattle, 15 goat and 4 dog isolates that carried
Stx1, Stx2 and eae genes (alone or in combination) were examined for the detection of EHEC O157:H7. Four isolates (50\%) from cattle and 1 (6.7\%) from a goat showed non-sorbitol-fermenting colonies. To confirm the presence of EHEC O157:H7, the suspected colonies were tested with Wellcollex E.coli. The results were negative for all isolates according to the kit directions.

The antimicrobial susceptibility profile of 233 E.coli isolates showed that the highest level of resistance was recorded against TE (53.5\%) followed by AMP (47.2\%), SXT (38.2\%), AMC (36.9\%), ENR (25.3\%) and EFT (17.6\%). Among 41 E.coli isolates that carried at least one virulence gene, 5 (12.2\%) were multi-drug resistant (MDR). The antimicrobial susceptibility results for virulent and avirulent E.coli isolates are shown in Table 3 and Table 4.

Table 2: The frequency and pathotypes of E.coli virulence genes in domestic animals

\begin{tabular}{|c|c|c|c|c|c|c|c|c|c|}
\hline Animal & Virulence genes & Pathotype & No of strains & & & & & & \\
\hline & & & & $S t x 1$ & $S t x 2$ & eae & $S T a$ & F41 & $\begin{array}{c}\text { F5 } \\
\text { (K99) }\end{array}$ \\
\hline \multirow[t]{4}{*}{ Cattle n:111 } & $S t x 1+e a e+S t x 2$ & STEC & $6(5.4 \%)$ & 6 & 6 & 6 & 0 & 0 & 0 \\
\hline & Stx $1+e a e$ & STEC & $1(0.9 \%)$ & 1 & 0 & 1 & 0 & 0 & 0 \\
\hline & $S T a$ & ETEC & $1(0.9 \%)$ & 0 & 0 & 0 & 1 & 0 & 0 \\
\hline & Eae & EPEC & $1(0.9 \%)$ & 1 & 0 & 0 & 0 & 0 & 0 \\
\hline Total & & & $9(8.1 \%)$ & $7(6.3 \%)$ & $6(5.4 \%)$ & $8(7.2 \%)$ & $1(0.9 \%)$ & 0 & 0 \\
\hline \multirow[t]{4}{*}{ Sheep n:25 } & Stx 1 & STEC & $4(16 \%)$ & 4 & 0 & 0 & 0 & 0 & 0 \\
\hline & Stx 2 & STEC & $3(12 \%)$ & 0 & 3 & 0 & 0 & 0 & 0 \\
\hline & $S t x 1+S t x 2$ & STEC & $3(12 \%)$ & 3 & 3 & 0 & 0 & 0 & 0 \\
\hline & $S T a$ & ETEC & $3(12 \%)$ & 0 & 0 & 0 & 3 & 0 & 0 \\
\hline Total & & & $13(52 \%)$ & $7(28 \%)$ & $6(24 \%)$ & 0 & $3(12 \%)$ & $\mathbf{0}$ & 0 \\
\hline \multirow[t]{3}{*}{ Goat $n: 45$} & Stx $x 1$ & STEC & $10(22.2 \%)$ & 10 & 0 & 0 & 0 & 0 & 0 \\
\hline & Stx 2 & STEC & $3(6.7 \%)$ & 0 & 3 & 0 & 0 & 0 & 0 \\
\hline & $S t x 1+S t x 2$ & STEC & $2(4.4 \%)$ & 2 & 2 & 0 & 0 & 0 & 0 \\
\hline Total & & & $15(33.3 \%)$ & $12(26.7 \%)$ & $5(11.1 \%)$ & 0 & 0 & $\mathbf{0}$ & 0 \\
\hline Dog n:35 & Eae & EPEC & $4(11.4 \%)$ & 0 & 0 & $4(11.4 \%)$ & 0 & 0 & 0 \\
\hline
\end{tabular}




\begin{tabular}{|c|c|c|c|c|c|c|}
\hline Cattle (111) & ENR $^{1}$ & $\mathbf{A M P}^{2}$ & $\mathbf{S X T}^{3}$ & EFT ${ }^{4}$ & $\mathbf{T E}^{5}$ & $\mathrm{AMC}^{6}$ \\
\hline $\mathbf{S}$ & $73(65.8 \%)$ & $35(31.5 \%)$ & $50(45 \%)$ & $77(69.4 \%)$ & $26(23.4 \%)$ & $55(49.5 \%)$ \\
\hline $\mathbf{R}$ & $38(34.2 \%)$ & $76(68.5 \%)$ & $61(55 \%)$ & $34(30.6 \%)$ & $85(76.6 \%)$ & $56(50.5 \%)$ \\
\hline Sheep (25) & ENR & $\mathbf{A M P}$ & SXT & EFT & TE & $\mathbf{A M C}$ \\
\hline $\mathbf{S}$ & $21(84 \%)$ & $18(72 \%)$ & $20(80 \%)$ & $25(100 \%)$ & $17(68 \%)$ & $18(72 \%)$ \\
\hline $\mathbf{R}$ & $4(16 \%)$ & $7(28 \%)$ & $5(20 \%)$ & $(0 \%)$ & $8(32 \%)$ & $7(28 \%)$ \\
\hline Goat (45) & ENR & AMP & SXT & EFT & TE & AMC \\
\hline $\mathbf{S}$ & $41(91.1 \%)$ & $38(84 \%)$ & $38(84 \%)$ & $42(93.3 \%)$ & $35(77.8 \%)$ & $40(88.9 \%)$ \\
\hline $\mathbf{R}$ & $4(8.9 \%)$ & $7(15.6 \%)$ & $7(15.6 \%)$ & $3(6.7 \%)$ & $10(22.2 \%)$ & $5(11.1 \%)$ \\
\hline Horse (4) & ENR & $\mathbf{A M P}$ & SXT & EFT & TE & AMC \\
\hline $\mathbf{S}$ & $4(100 \%)$ & $1(25 \%)$ & $2(50 \%)$ & $4(100 \%)$ & 0 & $3(75 \%)$ \\
\hline $\mathbf{R}$ & 0 & $3(75 \%)$ & $2(50 \%)$ & 0 & $4(100 \%)$ & $1(25 \%)$ \\
\hline Cat (13) & ENR & $\mathbf{A M P}$ & SXT & EFT & TE & $\mathbf{A M C}$ \\
\hline $\mathbf{S}$ & $8(61.5 \%)$ & $7(53.8 \%)$ & $9(69.2 \%)$ & $13(100 \%)$ & $6(46.2 \%)$ & $9(69.2 \%)$ \\
\hline $\mathbf{R}$ & $5(38.5 \%)$ & $6(46.2 \%)$ & $4(30.8 \%)$ & 0 & $7(53.8 \%)$ & $4(30.8 \%)$ \\
\hline Dog (35) & ENR & AMP & SXT & EFT & TE & AMC \\
\hline $\mathbf{S}$ & $27(77.1 \%)$ & $24(68.6 \%)$ & $25(71.4 \%)$ & $31(88.6 \%)$ & $20(57.1 \%)$ & $22(62.9 \%)$ \\
\hline $\mathbf{R}$ & $8(22.9 \%)$ & $11(31.4 \%)$ & $10(28.6 \%)$ & $4(11.4 \%)$ & $15(42.9 \%)$ & $13(37.1 \%)$ \\
\hline TOTAL & ENR & AMP & SXT & EFT & TE & AMC \\
\hline $\mathbf{S}$ & $174(74.7 \%)$ & $123(52.8 \%)$ & $144(61.8 \%)$ & $192(82.4 \%)$ & $108(46.4 \%)$ & $147(63.1 \%)$ \\
\hline $\mathbf{R}$ & $59(25.3 \%)$ & $110(47.2)$ & $89(38.2 \%)$ & $41(17.6 \%)$ & $125(53.6 \%)$ & $86(36.9 \%)$ \\
\hline
\end{tabular}

(1Enrofloxacin, 2Ampicillin, 3Trimethoprim/Sulfamethoxazole, 4Ceftiofur, 5Tetracyclines, 6Amoxycillin/ Clavulanic Acid) 
Table 4: Multi drug resistance pattern of virulence gene carrying E.coli isolates from animals

\begin{tabular}{|c|c|c|c|}
\hline Antimicrobial resistance profile & Number & & lence genes \\
\hline ENR - AMP - SXT- EFT - TE - AMC & 1 & A & Stx1, eae, Stx2 \\
\hline AMP - SXT - EFT - TE- AMC & 1 & A & Stx 1, eae, Stx2 \\
\hline AMP - SXT - TE - AMC & 1 & A & Stx $1, e a e$, St $x 2$ \\
\hline AMP - TE - AMC & 1 & A & Stx 1 , eae, Stx 2 \\
\hline AMP -SXT & 1 & A & Stx 1 , eae, Stx 2 \\
\hline AMP - TE & 1 & A & Stx 1 , eae, Stx 2 \\
\hline ENR - AMP - SXT - TE - AMC & 1 & A & STa \\
\hline \multirow{5}{*}{ AMC } & \multirow{5}{*}{5} & B & Stx 1, Stx 2 \\
\hline & & B & Stx 1, Stx 2 \\
\hline & & B & Stx 1 \\
\hline & & $\mathrm{C}$ & St $x 2$ \\
\hline & & $\mathrm{D}$ & eae \\
\hline TE & 1 & A & Stx 1 , eae \\
\hline
\end{tabular}

\section{Discussion}

Phenotypic screening techniques for the detection of E.coli pathotypes other than STEC are not adequate. Molecular methods have been used by several clinical laboratories to facilitate the identification of organisms that cannot be cultivated due to unusual growth characteristics or antibiotic treatment, or that cannot be classified by phenotypical methods. This study evaluated the major virulence genes of fecal E.coli isolates from variety of domestic animals in Turkey. In the present study, DEC represented $17.6 \%$ (41/233) of isolates, including Stx1 (11.1\%), Stx2 (7.3\%), eae $(5.2 \%)$ and STa $(1.7 \%)$ of isolates from cattle. Several researchers reported similar results, with Stx1, eae and Stx 2 in combination or as separate genes. ${ }^{12,13}$ Several studies have revealed that strains possessing only Stx2 exhibited potentially more virulence than strains possessing Stx 1 or Stx 1 and Stx $2 .{ }^{3}$ This study revealed that $2.5 \%$ of isolates from sheep and goats carried the more virulent Stx2 gene. Because sheep and goats are an important source of protein in Turkey, the presence of the Stx2 gene in E.coli isolates from small ruminants could be therefore a potential source of community-associated human HUS infection.

In the present study, the detection rate of the eae gene in STEC cattle isolates was 7.2\% (n:7) whereas Nguyen et al. ${ }^{14}$ reported that it was $9.8 \%$. Barett et al. ${ }^{15}$ reported that eae may be necessary for the expression of full virulence of STEC for humans, demonstrating a potential risk for zoonotic infections. These results showed that eae carriage by cattle in Turkey should be considered an important zoonotic threat for STEC transmission between humans and animals.

As shown in this study, STEC has been detected at higher rates in sheep (40\%) than in cattle (7.2\%). This finding is similar to Beutin et al. ${ }^{16}$, who recorded a prevalence of $66.6 \%$ in sheep and $21.1 \%$ in cattle. The difference between the two species may be due to the small number of animals tested. These results indicate that sheep may be a primary source of STEC.

Fecal carriage of EHEC O157 in farm animals has been reported in several countries. The presence of EHEC O157:H7 was tested in this study, and it was not detected in any isolates. These findings were consistent with a study by Leomil et al. ${ }^{17}$ who also observed that all of the tested isolates were negative for EHEC O157:H7.

In the present study, ETEC was detected at a low frequency $(0.9 \%)$ in cattle isolates. Similar results were reported by Blanco et al. ${ }^{18}$ who detected ETEC in a single strain. As demonstrated in the present study, sheep ETEC represented $12 \%$ of the isolates, which was approximately in agreement with the result of $11.2 \%$ reported by Turkyilmaz et al. ${ }^{19}$. All isolates tested in this study were negative for F41 and F5 (K99) genes. These findings were in agreement with the results of the study by DeVerdier et al. ${ }^{20}$, who reported that F5 was not observed in any tested isolates. The health status of the animals was not considered for this study, which may be a reason for of the lack of evidence of fimbrial F41 and F5 (K99) genes in the samples tested.

In the present study, EPEC was detected in $0.9 \%$ of cattle, which agreed with Holland et al. ${ }^{21}$, who reported that cattle can be considered as a main reservoir for EPEC. Recent findings have shown that the transmission of DEC strains occurs between pet animals and humans. ${ }^{22}$ The zoonotic importance of EPEC was demonstrated by Beutin ${ }^{23}$, who described it in isolates from dogs. In the present study, EPEC was detected in $11.4 \%$ of dogs and was not detected in cats. These results agreed with those of Puno-Sarmiento et al. ${ }^{24}$, who reported that all of the cat isolates were negative, and a portion of dog isolates were positive for EPEC. In the present study, the antimicrobial susceptibility profile showed that the highest resistance recorded was against tetracycline in all animals. These findings were consistent with those of several researchers who reported that the resistance was most frequent for sulfonamides, tetracycline, and streptomycin in domestic animals. ${ }^{25,}{ }^{26}$ In the present study, among all E.coli isolates that carried at least one virulence gene, $12.2 \%$ were MDR, $4.9 \%$ were resistant to two antibiotics, $14.6 \%$ were resistant to one antibiotic and $68.3 \%$ were susceptible to all antibiotics. Enterobacteriaceae commonly carry multiple large plasmids, a number of which can contain resistance genes to 10 or more antimicrobial agents. ${ }^{27}$ As shown in this study, the highest degree of MDR was seen in strains that carried 3 virulence genes in combination. These results suggest that a high prevalence of virulence factors might be related to a high MDR 
profile.

\section{Conclusions}

In conclusion, virulence factors Stx1, Stx2, eae and STa, which indicate the presence of pathogenic E.coli, were detected by multiplex PCR methods from a variety of animals in Turkey. The results of this study suggested that cattle, sheep, goats, and dogs are potential reservoirs of STEC, ETEC and EPEC for humans. A high MDR profile was observed among pathogenic E.coli strains. The presence of virulent E.coli isolates from domestic animals and their antimicrobial resistance pattern should be described by further epidemiological methods for understanding of zoonotic significance by potential transmission of E.coli.

\section{Funding}

This research was supported by Uludag University Scientific Research Projects KUAP (V) 2014/43 entitled 'Determination of some virulence genes of Escherichia coli isolates from animals by multiplex PCR' and a part of the doctorate thesis (2017) of Mohammed Khider entitled' PCR Detection of some virulence genes in Escherichia coli isolates obtained from animal sources'.

Declarations of interest

None.

\section{References}

1. Strockbine N.A, Bopp C.A., Fields P.I., Kaper J.B., Nataro J.P. Chapter 37. Esherichia, Shigella and Salmonella. In Jorgensen JH, ed. Manual of Clinical Microbiology. Volume 1.11th edition, ASM Press, Washington, DC; 2015, 685-713.

2. Gyles CL: Shiga toxin-producing Escherichia coli: an overview. J. Anim. Sci. 2007;85 (13), 45-62.

3. Nataro JP, Kaper JB: Diarrheagenic Escherichia coli. Clin. Microbiol. Rev. 1998; 11,142-201. doi: 10.1128/ CMR.11.1.142

4. Gyles CL, Fairbrother JM : Escherichia coli, in: Gyles, C. L., Prescott, J. F., Songer, J. G. and Thoen, C.O. (eds), Pathogenesis of Bacterial Infections in Animals.4th ed.Wiley-Blackwell, Ames, IA, U.S.A,; 2010, 267-308.

5. Kaper JB, Nataro JP, Mobley HL. Pathogenic Escherichia coli. Nat. Rev. Microbiol. 2004; 2(2), 123-140.

6. Law D: Virulence factors of Escherichia coli O157 and other shiga toxin-producing E.coli. J. App. Microbiol. 2000; 88(5): 729-745.

7. Blanco M, Blanco J, Blanco JE, et al. Enterotoxigenic, verotoxigenic, and necrotoxigenic Escherichia coli isolated from cattle in Spain. Am. J. Vet. Res. 1993; 54 (9): 1446-1451.

8. Pitout, JD, Laupland KB. Extended-spectrum beta-lac- tamase-producing Enterobacteriaceae: an emerging public health concern. Lancet Infect. Dis. 2008; 8(3): 159-166. doi: 10.1016/S1473-3099(08)70041-0.

9. Sawant AA, Hegde NV, Straley BA, et al. Antimicrobial-Resistant Enteric Bacteria from Dairy Cattle. App. Environ.Microbiol, 2007; 73, 156-163. DOI: 10.1128/ AEM.01551-06.

10. Franck SM, Bosworth BT, Moon HW. Multiplex PCR for enterotoxigenic,

attaching and effacing, and Shiga toxin- producing Escherichia coli strains from calves. J. Clin. Microbiol.,1998; 36(6), 1795-1797.

11. Anonymous: European Committee on Antimicrobial Susceptibility Testing Breakpoint tables for interpretation of MICs and zone diameters Version 7.1, valid from 2017-03-10 http://www.eucast.org/fileadmin/src/media/PDFs/EUCAST_files/Breakpoint_tables/v_7.1_Breakpoint_Tables.pdf.(12.19.2017)

12. Guler L, Gunduz K, Ok U (: Virulence Factors and Antimicrobial Susceptibility of

Escherichia coli Isolated from Calves in Turkey. Zoonoz. Pub. Health, 200855 (5), 249-257, https://doi. org/10.1111/j.1863-2378.2008.01121.x

13. Galli L, Miliwebsky E., Irino K, et al. Virulence profile comparison between LEE-negative Shiga toxin-producing Escherichia coli (STEC) strains isolated from cattle and humans. Vet. Microbiol., 2010: 143(2-4); 307-313, doi: 10.1016/j.vetmic.2009.11.028.

14. Nguyen TD, Vo TT, Vu-Khac H. Virulence factors in Escherichia coli isolated

from calves with diarrhea in Vietnam. J. Vet. Sci. 2011:12 (2); 159-164

Barett T J, Kaper JB, Jerse A E, et al. Virulence factors in shiga-like toxin producing E.coli isolated from humans and cattle. J. Infect. Dis. 1992: 165(5), 979-980, DOI:10.1093/infdis/165.5.979.

15. Beutin L, Geier D, Steinruck H, et al. Prevalence and Some Properties of Verotoxin (Shiga-Like Toxin)-Producing Escherichia coli in Seven Different Species of Healthy Domestic Animals. J. Clin. Microbiol. 1993; 31(9): 2483-2488.

16. Leomil L, Aidar-Ugrinovich L, Guth BE, et al. Frequency of Shiga toxin-producing Escherichia coli (STEC) isolates among diarrheic and non-diarrheic calves in Brazil. Vet. Microbiol. 2003;97(1-2),103-109.

17. Blanco J, Gonzalez EA, Garcia S. Production of toxins by Escherichia coli

strains isolated from calves with diarrhea in Galicia (north-western Spain). Vet. Microbiol, 1988; 18(34):297-311.

18. Turkyilmaz S, Eskiizmirliler S, Tunaligil S, et al.Identi- 
fication, characterization and molecular epidemiology of Escherichia coli isolated from lamb and goat kids with diarrhea. Acta Vet. Brno.2013; 82(4):357-362. DOI: 10.2754/avb201382040357

19. De Verdier K, Nyman A, Greko C. et al. Antimicrobial resistance and virulence factors in Escherichia coli from Swedish dairy calves. Acta Vet. Scand. 2012; 54(2), doi: 10.1186/1751-0147-54-2.

20. Holland R E, Wilson RA, Holland M S, et al. Characterization of eae+ Escherichia coli isolated from healthy and diarrhoeic calves. Vet. Microbiol., 1999; 66(4): 251-263.

21. Khakhria R, Duck D, Lior H. Extended phage typing scheme for Escherichia coli 0157:H7. Epidemiol Infec., 1990; 105 (3)511-520. DOI: https://doi.org/10.1017/ S0950268800048135

22. Beutin L. Escherichia coli as a pathogen in dogs and cats. Vet. Res. 1999; 30(2-3): 285- 298.

23. Puno-Sarmiento J, Medeiros L, Chiconi C. et al. Detection of diarrheagenic Escherichia coli strains isolated from dogs and cats in Brazil. Vet. Microbiol. 2013; 166(3-4): 676-680, https://doi.org/10.1016/j.vetmic.2013.07.007.

24. Bryan A, Shapir N, Sadowsk MJ. Frequency and Distribution of Tetracycline resistance genes in genetically diverse, non selected, and non clinical Escherichia coli strains isolated from diverse human and animal sources. App. Environ. Microbiol., 2004; 70(4) , 25032507.

25. Talukdar PK, Rahman M, Rahman M, et al. Antimicrobial resistance, virulence factors and genetic diversity of Escherichia coli isolates from household water supply in Dhaka, Bangladesh. PLoS ONE. 2013; 8, e61090. doi: 10.1371/journal.pone.0061090.

26. Jacoby GA, Archer GL. New mechanisms of bacterial resistance to antimicrobial agents. New Eng. J Med. 1991; 324: 601- 612. DOI: 10.1056/ NEJM199102283240906 